\title{
Better emotion modeling by taking into account environment factors and human profiles in crisis situations
}

\author{
Azise Oumar DIALLO ${ }^{1}$, Dominique LONGIN $^{2}$, HO Tuong Vinh ${ }^{1,3}$ \\ ${ }^{1}$ IFI, Vietnam National University in Hanoi, Vietnam \\ ${ }^{2}$ CNRS-IRIT, Toulouse University \\ ${ }^{3}$ Sorbonne Université, IRD, UMMISCO, F-93143, Bondy, France \\ Email: diallo.p21@ifi.edu.vn, Dominique.Longin@irit.fr, ho.tuong.vinh@ifi.edu.vn
}

\begin{abstract}
Crisis investigation reports revealed a gap between existing security measures and the actual behaviour of victims, especially related to their emotional states. We propose a new mathematical model of emotion for simulating human behaviours in crisis situations. The model takes into account various environmental situations (static or dynamic) and the profiles of the people involved. It is more close to findings in psycho-physiological studies of emotion and allows to make the evacuation simulation more realistic. The entire theoretical model has been implemented and evaluated in the IDE R-Studio and the agent-based GAMA simulation platform. The obtained results outperform the results presented in the related work.
\end{abstract}

Index Terms-emotion, evacuation, agent-based model

\section{INTRODUCTION}

Emotions are more and more investigated in social and computational sciences. Facing danger situations, man human can present several emotional states (worry, anxiety, fear, despair, panic and terror). Several works on fear show that a frequent reaction is cooperative action (an individual who is little affected by events actively helps others). In this regard, reference may be made to the sociological works of Quarantelli [5], [6] or more recently Drury and al. on largescale disasters or terrorist attacks (see [1]-[3] for example). Contrary to what we might think, people rarely panic but adopt social behaviours (people are naturally social).

In this work, we focus on analyzing the different levels of fear per population in an emergency situation. We seek precisely to study the conditions of triggering of this emotion according to the variability of the environment and the different profiles of people. We propose a new mathematical model of emotion that takes into account various environmental situations (static or dynamic) and the profiles of the people involved.

The remainder of the paper is organized as follows. In section II, we present the related work on the influence of the environment on emotion. In section III, we present a detailed description of our improved model. In section IV, we detailed the experimental setup and we present obtained results. Finally, in section V, we conclude the paper and we highlight some perspectives for future work.

\section{EXISTING Models OF THE INFLUENCE OF THE ENVIRONMENT ON EMOTION}

As far as we know, only few works are concerned by the role played by the environment on emotion. In our previous works [8], [9], the intensity of emotions are dynamically computed by taking three factors into account: decay, environment and contagion.

In those models, the environment influence on the $i$ 's fear intensity level is as follows:

$$
\text { fearEnv }(t)=\frac{1}{1+e^{-\lambda_{i}\left(1-\frac{d(t)}{v(t)}\right)}} .
$$

where $\lambda_{i}$ represents the perceived value of the dangerousness of the environment by the agent $i$ and fearEnv $(t)$ the intensity of the fear generated by the $\lambda_{i}$ at time $t$.

We can see that this function mainly suffer from two problems: its dependence with the variable $t$ (which should not be the case) and "a lack of coherence" (intuitively speaking) (when $\lambda_{i}=0$ (i.e., no danger detected) then fearEnv $(t)=$ $\frac{1}{1+1}=0.5$ (on a scale of 1 ), even though it is expected that, in this case, the agent feels no fear.

\section{IMPROVED MODEL OF ENVIRONMENT INFLUENCE ON EMOTION}

\section{A. Study of environment influence function on emotion}

We propose first of all to state some number of postulates that this function must intuitively satisfy.

Postulate 1 (input variable): Logically, fearEnv should not directly depend on the variable $t$ (i.e., the time).

Indeed, time is not here an intrinsic variable attached to the level intensity of fear: it plays only role when the dangerousness of the environment varies over time. It is therefore the function which calculates the dangerousness of the environment that should depend on time, whereas fearEnv should only depend on this danger $\lambda_{i}$ (see the section III-B for the definition of this function). In particular, if it is constant over time, the intensity of the emotion should be as constant. Of course as specified in [8], the intensity of the emotion decreases over a period of time (decay factor). 


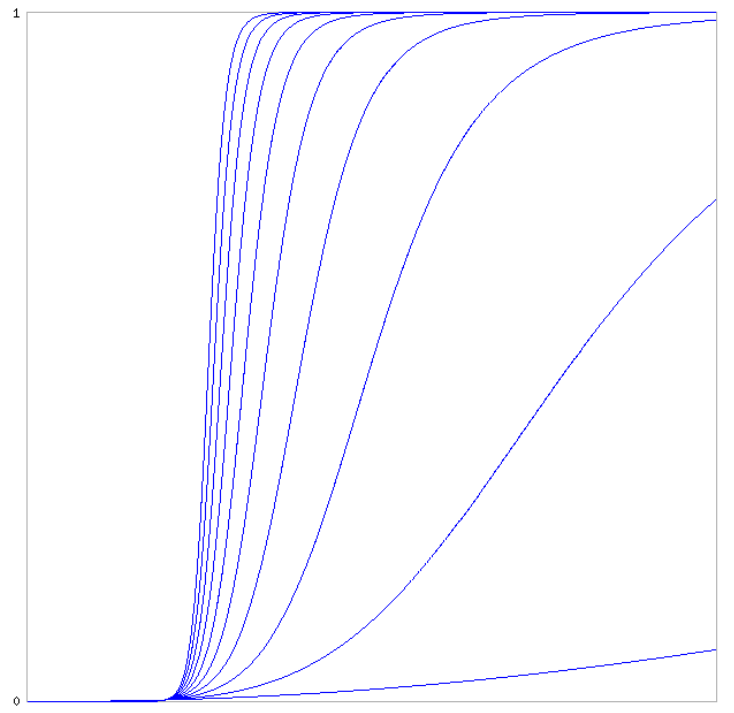

Figure 1. Example of graphical representations of the intensity of fear function of 10 agent profiles with different levels of familiarity to danger: dangerFam varies from 0 to 0.9 with a step of 0.1 and corresponds to the curve from left to right. These representations have been performed on the online platform "MAFA Function Plotter".

Postulate 2 (domain): The function fearEnv should be defined such as:

$$
\text { fearEnv }:[0, m] \longrightarrow[0,1]
$$

where $m \in \mathbb{R}^{+}$and such that $\lim _{x \rightarrow 0^{+}} \operatorname{fearEnv}(x)=0$ and $\lim _{x \rightarrow m} \operatorname{fearEnv}(x)=1$.

We focus only on the interval where the dangerousness of the environment is positive. We normalize the intensity level of fear between 0 and 1 to make it more convenient to handle and to compare, etc. The previous postulate also induces the idea that in the absence of danger, the level of intensity of the fear is zero, whereas it is highest when the level of dangerousness of the physical environment is the maximum.

Postulate 3 ("toggle" effect): The intensity of an emotion corresponds mathematically to a sigmoid type function, i.e. an increasing function where this increasing is very fast over some limited interval [4], [7].

Postulate 4 (familiarity with danger): Each agent may be more or less familiar with the danger that surrounds him/her, and it is necessary to introduce a familiarity factor to the danger that will be noted dangerFam.

Someone familiar with some type of danger will have emotional levels that will increase less strongly and occur ("toggle" effect) less quickly than for other agents (see Figure 1).

In considering all the postulates mentioned above, this function must have the general shape of a sigmoid as specified in [8] which can be defined as follows:

$$
\text { fearEnv }(x)=\frac{1}{1+e^{(\epsilon-(1-\text { dangerFam }) \kappa \log (x))}}
$$

where $\epsilon$ and $\kappa$ are the calibration coefficients of the curve.
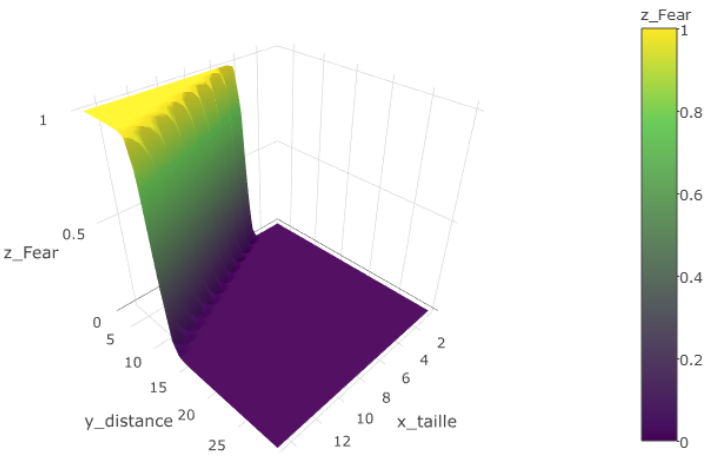

Figure 2. Example of graphical representation of the intensity of fear function according to a focus of danger and its distance from the agent with dangerFam $=0.5$. This representation has been performed by the integrated development environment (IDE) for R: RStudio.

The Figure 1 provides the graphical (sigmoidal) representations of this function for 10 user profiles with different danger familiarity coefficients (dangerFam). For indication, here $\epsilon=6, \kappa=50$. The abscissa represents the dangerousness of the environment (we specify in the next section how it is calculated) and on the ordinate the level of fear of the corresponding agent. The "toggle" effect of sigmoid is the moment when the agent moves from a state where he does not yet feel the emotion to a state where he begins to perceive it.

The Figure 2 illustrates the impact of one fire and of its distance on the initial fear level for an agent given with dangerFam $=0.5$ with R-Studio.

Thus, we find that the more an agent is familiar with a danger (i.e., more dangerFam is close to 1 ), the more the curve representing the evolution of its fear according to the dangerousness of the environment is flattened. Conversely, when familiarity with danger is low, this curve increases very quickly, meaning that the level of fear increases quickly (and that the fear emotion is triggered quickly). The intensity level of fear stabilizes as a result of the "toggle" effect.

\section{B. Definition of the function of dangerousness of the environ- ment dangerEnv}

This function must also respect some postulates.

Postulate 5 (input variable): The level of danger of the environment is a priori proportional to the number of foci of danger and their size, and inversely proportional to their distance from the agent considered.

Definition 1 (intrinsic dangerousness): We call intrinsic dangerousness of a danger for a given agent, the ratio of its size $\rho$ to its distance $\delta>0$ from this observer, formally: $\frac{\rho}{\delta}$.

Nevertheless, we know that the more danger foci are numerous, and the less the addition of a new focus of danger is perceived as dangerous. We choose here a simple mechanism: the first danger will be taken into account according to the 
entirety of its intrinsic danger, the second half of its intrinsic danger, the third for a third, etc. This leads to a logarithmic increasing depending to the number of danger foci.

Definition 2 (objective dangerousness of an element of the environment): Let $\rho_{i}$ be the size of the danger ${ }^{1}$ number $i^{2}$ and $\delta_{i}$ its distance from the agent, so the objective danger of $i^{e}$ element of the environment (noted : dangerousness ${ }_{i}$ ) is defined as :

$$
\text { dangerousness }_{i}= \begin{cases}\frac{\rho_{i}}{i \times \delta_{i}} & \text { when } i>0 \\ 0 & \text { else }\end{cases}
$$

where $\rho_{i}$ and $\delta_{i}$ are assumed to be characteristic of the danger observed at a given moment (dependent variables of the modeled system and the moment of observation).

Definition 3 (objective dangerousness of the environment): The objective dangerousness of the environment can then be captured by:

$$
\text { dangerEnv }=\sum_{i \in[1 . . N]} \text { dangerousness }_{i}
$$

where $N$ is the total number of dangers in the environment.

Of course, the order in which danger is apprehended directly influences the ratio with that it is taken into account in calculating its objective danger. Indeed, if for example we consider a big fire $\rho_{b}$ and a small fire $\rho_{s}$ located at the same distance $\delta$, the objective dangerousness of the environment will be greater if we consider the entirety of the objective dangerousness of the big fire and half of the objective dangerousness of the small fire, rather than if we consider the entirety of the small fire and half of bigger. Formally: $\frac{\rho_{b}}{\delta}+\frac{\rho_{s}}{2 \times \delta}>\frac{\rho_{s}}{\delta}+\frac{\rho_{b}}{2 \times \delta}$ when $\rho_{b}>\rho_{s}$.

Postulate 6 (subjectivity of perception of danger): The level of dangerousness perceived ( $v s$ real) by the agent is a subjective characteristic of the agent that must be taken into account and will be represented as a coefficient percepSubjectivity. This coefficient being an intrinsic characteristic of the agent and assumed to be constant.

Definition 4 (subjective dangerousness): For any given agent $i$ characterized by a coefficient of subjective perception of danger percepSubjectivity ${ }_{i}$, the subjective danger to the agent $i$ of the environment is defined by:

$$
\text { dangerEnv }_{i}=\text { percep Subjectivity }_{i} \times \text { dangerEnv }
$$

In what follows, we represent the dangerousness function as defined above by varying the coefficient of subjective


shown in the Figure 3. We have taken the example of 10 fireplaces numbered from 1 to 10 , of the same size $(1 \mathrm{~m})$, and

\footnotetext{
${ }^{1}$ In the case of fires for example, we can assimilate this size to the diameter of the fires considered.

${ }^{2}$ This number corresponds to the serial number in which the fires are taken into account. This number depends on the function of aggregation of the fires that we have chosen: according to for example that we take into account the fires as and when they appear, or according to their decreasing size, the value dangerousness $_{i}$ will be different.
}

located respectively $1 \mathrm{~m}, 2 \mathrm{~m}, 3 \mathrm{~m}, 5 \mathrm{~m}, 7 \mathrm{~m}, 11 \mathrm{~m}, 13 \mathrm{~m}, 17 \mathrm{~m}$, $23 \mathrm{~m}$ and $27 \mathrm{~m}$ from the agent.

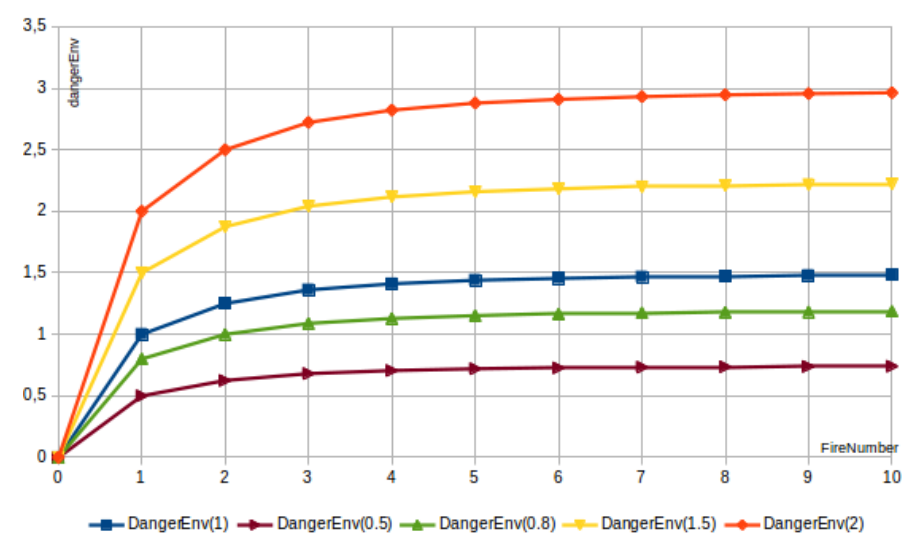

Figure 3. Example of graphical representation of the function of the level of dangerousness of the physical environment

We can see that when percepSubjectivity $=1$ (blue curve (DangerEnv(1))) the agent has a perception exactly corresponding to the objective dangerousness of the environment. The curves below the blue curve (DangerEnv(0.5) and DangerEnv(0.8)) correspond to "optimistic" individuals (underestimating the dangerousness of the situation). Finally, the curves situated above (DangerEnv(1.5) and DangerEnv(2)), correspond to "pessimistic" agents (or very fearful) tending to overestimate the danger surrounding.

We formulate the algorithm for the computation of the subjective dangerousness as following:

1) determine the fire order function (by increasing / decreasing intrinsic dangerousness, or by order of appearance, etc.) ;

2) at each step of the simulation:

- observe the environment and perceive the dangers inside it;

- assign to each fire a serial number (according to the order function chosen in 1));

- compute the subjective dangerousness of the environment.

\section{Agent-based Simulation}

In our simulation, we consider that the foci of danger are fires. We assume that a fire is propagated in a random direction at each step of the simulation. While spreading, a fire burns any other agents (tree, habitat, human agent) in its location. Finally, if the fire returns to a position it has already burned, it turns off.

There are different types of agents in our model: elements of the environment (trees, buildings, shelters, fire, wind) and humans.

1) Environment: The environment is a area of $100 \mathrm{~m} \times 100 \mathrm{~m}$ and contains different types of agents: fire, trees, buildings, shelters and inhabitants. Each agent is randomly located in the environment. 
2) Human: Human agents are represented by six (06) agent profiles representing their familiarity with danger (dangerFam) and their perception of the dangerousness of the environment (percepSubjectivity). These profiles are:

- The fearful and inexperienced inhabitant: This agent profile represents a pessimistic inhabitant, fearful and unfamiliar with the danger.

- The fearful and experienced inhabitant: This agent profile represents a pessimistic inhabitant, fearful and familiar with the danger.

- The optimistic and inexperienced inhabitant: This agent profile represents an inhabitant who underestimates the danger and is unfamiliar with the danger.

- The optimistic and experienced inhabitant: This group is an experienced agent who perceives the environment as not very dangerous compared to its objective danger.

- The objective and inexperienced inhabitant: It is an agent who has an objective perception of the danger of the environment and not experienced.

- The objective and experienced inhabitant: It is an agent who has an objective perception of the dangerousness of the environment and experienced.

\section{EXPERIMENTAL RESULTS}

This section presents the experiments of our model implemented in GAMA agent-based simulation platform.

Two test scenarios defined as follows: environment without fire spread and environment with fire spread. To do that, we chose to simulate bushfires to allow us to observe the triggerings of fear and the evolution of its intensity in different populations of human according to different configurations of the environment (number, size, and position of the fire). In addition, it allows to involve several profiles of more or less experienced people and to change the dangerousness of the environment by spreading fire through trees and habitats. It should be noted that in each scenario, the number of inhabitants does not change. We have 10 individuals for each profile, i.e. 60 individuals. In addition, we arranged the individuals of each profile in the same location chosen randomly and not necessarily in the buildings. Precisely, we have 10 different locations and in each location there are 6 individuals corresponding to each profile.

The Figure 4 gives an overview of the simulation environment with 25 trees whose 5 are on fire, 10 buildings and 10 inhabitants for each profile in 10 different locations.

\section{A. Environment without fire spread}

In this scenario, our model is simulated in environment without fire spread where the number of fires remains constant throughout the simulation. However, we vary the sizes and the distances of the fires from the inhabitants.

The Figure 5 and 6 give respectively an insight into the evolution of subjective dangerousness and intensity of fear for each agent profile with 50 fires.

We can see that after the detection of fires, the dangerousness of the environment and the intensity of fear remain

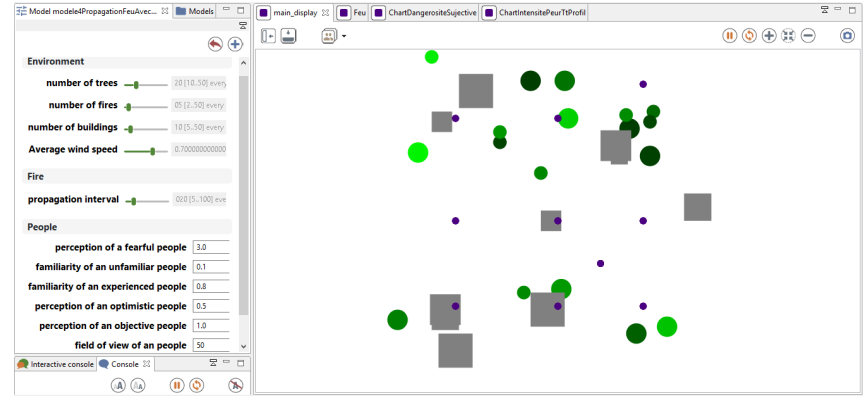

Figure 4. Environment with 20 tree agents (green discs), 10 buildings (gray squares) and 60 inhabitants (blue dots) divided into 10 different zones of 6 individuals of different profiles.

constant for all inhabitants. This is mainly due to the fact that there is no propagation (evolution) of fire in the environment. Moreover, the dangerousness of the environment and the intensity of the fear vary according to the profile of the inhabitants. These first results allow us to classify the different profiles of individuals according to their sensitivity to the danger. Thus, unsurprisingly, we realize that scared individuals are the most sensitive (orange curves on the figures, which are the highest). Then, optimistic individuals (yellow curves) always tend to underestimate the danger compared to reality (objective individuals, indigo curves).

Perhaps one of the crucial questions was: how do the intensities of fear evolve for fearful and familiar individuals to danger and objective and unfamiliar ones to danger? The results obtained indicate that it is the fearful individuals who are always the most sensitive to danger. We can then deduce that the perception of danger is stronger than the familiarity with it.

\section{B. Environment with fire spread}

In this case, the model is simulated in a dynamic environment (the fire can spread causing an increase in the number of foci of danger and a variation in their distance from the agents). This scenario therefore required the implementation of a fire propagation model, making the previous model even more realistic. The implemented propagation model, although simple, takes into account all the elements involved in the propagation of fire such as the wind, the nature of the burnt surface, etc. The Figure 7 and 8 give respectively an insight into the evolution of subjective dangerousness and intensity of fear for each agent profile with 10 initial fires.

By analyzing these results, we see that, the intensities of fear evolve according to the dangerousness of the environment. In particular, the intensity of fear is correlated with the number of dangers. These results make it possible to highlight the variation of the emotion as a function of time. In the first scenario where the environment did not evolve, the intensity of the emotion remained constant. In addition, this allows to answer one of the main problems related to the function of the influence of the environment on the emotion of [8]. Moreover, each profile has a maximum value of the intensity of the fear. 


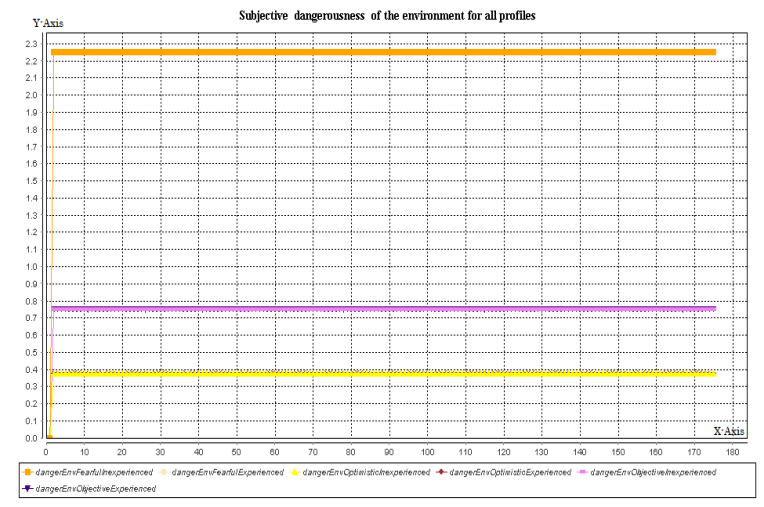

Figure 5. Evolution of the subjective dangerousness of the environment (Y-Axis) as a function of time (simulation cycle, X-Axis) by profile of scenario 1 with 50 foci fires

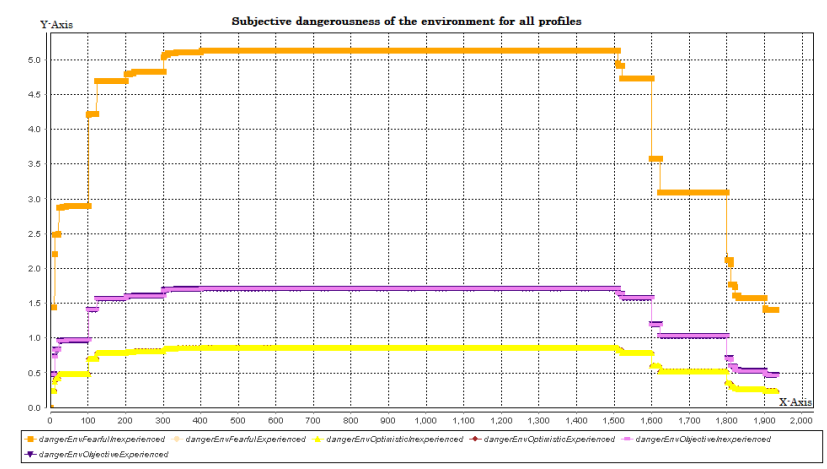

Figure 7. Evolution of the subjective dangerousness of the environment (Y-Axis) as a function of time (simulation cycle, X-Axis) by profile of scenario 2 with 10 initial foci fires

Once this value is reached, it no longer changes and no matter the number of foci of danger. Our function is very faithful to the pace defined by the authors of [8] who address of the sigmoidal aspect of the curve.

\section{CONCLUSION}

In this paper, we proposed an improved mathematical model of the influence of environment on emotions that is presented in [8]. The obtained equations help to better represent the conditions of triggering fear in a variable environment.

Moreover, we identified credible and intuitive parameters in order to be able to use our simulation in a predictive way, that means, for a given agent population with particular characteristics, we can predict what will be their emotional behavior in a situation of fear. From there, we can extrapolate solutions to improve the survival rate of people in a given crisis situation. This last part constitutes a middle or long term perspective of our work.

In some considerations, our approach may seem a little subjective in the sense that the values of the parameters we used are not derived from real situations (investigation reports during a crisis, field surveys, surveys of people...). Thus, in the short term, we plan to carry out a survey of people according



Figure 6. Evolution of the intensity of fear (Y-Axis) as a function of time (simulation cycle, $\mathrm{X}$-Axis) for each profile of scenario 1 with 50 foci fire

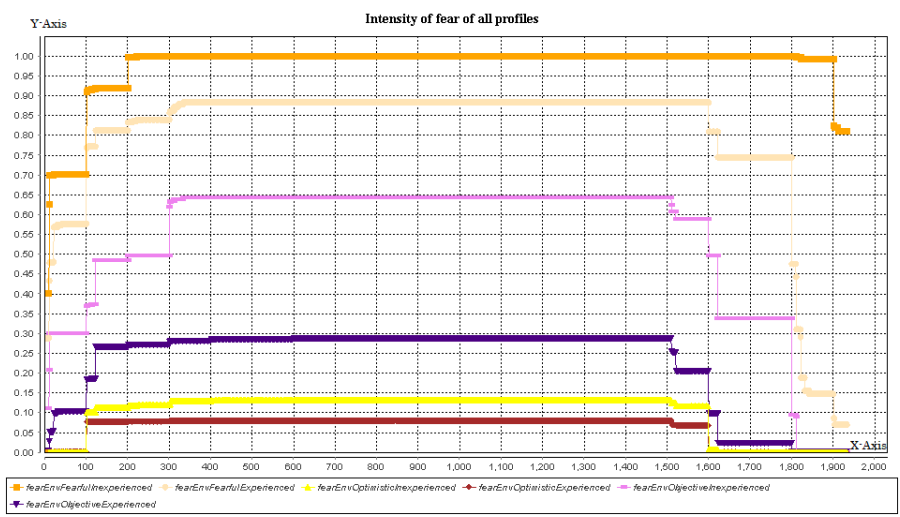

Figure 8. Evolution of the intensity of fear (Y-Axis) as a function of time (simulation cycle, X-Axis) for each profile scenario 2 with 10 initial foci fires

to different scenarios in order to have more objective values to parameterize the agents. Thus, we will be able to better evaluate our model and consequently improve it if necessary.

\section{REFERENCES}

[1] J. Drury and C. Cocking. The mass psychology of disasters and emergency evacuations: A research report and implications for practice. Technical report, Department of Psychology, University of Sussex, 2007.

[2] J. Drury, C. Cocking, and S. Reicher. Everyone for themselves? a comparative study of crowd solidarity among emergency survivors. British Journal of Social Psychology, 48:487-506, 2009.

[3] John Drury, Chris Cocking, and Steve Reicher. The nature of collective resilience: Survivor reactions to the 2005 london bombings. International Journal of Mass Emergencies and Disasters, 27(1):66-95, 2009.

[4] R. Lazarus. Emotion and Adaptation. Oxford University Press, 1991.

[5] E. Quarantelli. The nature and condition of panic. American Journal of Sociology, pages 267-275, 1954.

[6] E.L. Quarantelli. The sociology of panic. In Smelser and Baltes, editors, International Encyclopedia of the Social and Behavioural Sciences, pages 11020-11023. Pergamon Press, New York, 2001.

[7] D. Sander and K.R. Scherer, editors. Traité de psychologie des émotions. Dunod, $2^{\mathrm{e}}$ edition, 2014.

[8] X.H. Ta, B. Gaudou, D. Longin, E. Amouroux, and T.V. Ho. Emotion in the evacuation process: Formal model and simulation. In Proceedings of the Seventh Symposium on Information and Communication Technology, SoICT '16, pages 107-114, New York, NY, USA, 2016. ACM.

[9] Xuan-Hien Ta, Benoit Gaudou, Dominique Longin, and Tuong Vinh Ho. Emotional contagion model for group evacuation simulation. Informatica, 41(2):169-182, 2017. 